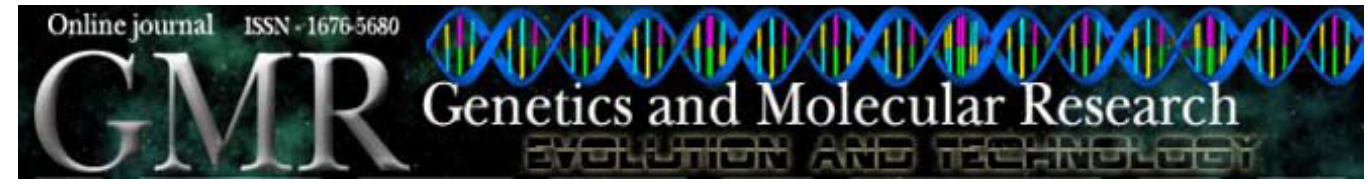

\title{
Allele frequencies for 12 autosomal short tandem repeat loci in two Bolivian populations
}

\author{
L. Cifuentes ${ }^{1}$, H. Jorquera ${ }^{2}$, M. Acuña ${ }^{1}$, J. Ordóñez ${ }^{2}$ and A.L. Sierra ${ }^{4}$ \\ ${ }^{1}$ Programa de Genética Humana, ICBM, Facultad de Medicina, \\ Universidad de Chile, Santiago, Chile \\ ${ }^{2}$ Genética y Tecnología Ltda., Santiago, Chile \\ ${ }^{3}$ Laboratorio Gen y Vida, La Paz, Bolivia \\ ${ }^{4}$ Laboratorio de Análisis Clínico Dr. Zuna Ltda., Santa Cruz, Bolivia \\ Corresponding author: L. Cifuentes \\ E-mail: 1cifuent@med.uchile.cl
}

Genet. Mol. Res. 7 (1): 271-275 (2008)

Received December 20, 2007

Accepted January 27, 2008

Published March 25, 2008

\begin{abstract}
Two hundred and sixty unrelated subjects who asked for paternity testing at two Bolivian Laboratories in La Paz and Santa Cruz were studied. The loci D3S1358, vWA, FGA, D8S1179, D21S11, D18S51, D5S818, D13S317, D7S820, TH01, TPOX, and CSF1PO were typed from blood samples, amplifying DNA by polymerase chain reactions and electrophoresis. Allele frequencies were estimated by simple counting and the unbiased heterozygosity was calculated. Hardy-Weinberg equilibrium was studied and gene frequencies were compared between the two samples. All loci conformed to the Hardy-Weinberg law and allele frequencies were similar in samples from the two cities. The Bolivian gene frequencies estimated were significantly different from those described for Chile and the United States Hispanic-Americans for most of the loci.
\end{abstract}

Key words: DNA typing; Population genetics; Short tandem repeat; Allele frequencies; Forensic science; Bolivian population 


\section{INTRODUCTION}

The usefulness of genetic markers for identity testing and paternity analysis is based on known allele frequencies for the genetic markers analyzed. Yet, there is no information published about gene frequencies of multiallelic loci in the Bolivian population.

The current Bolivian population arose from the admixture between aborigine populations of Mongoloid origin (Amerindians) and Spanish conquerors of Caucasian origin.

The present study describes the allele frequencies for twelve short tandem repeat (STR) loci (D3S1358, vWA, FGA, D8S1179, D21S11, D18S51, D5S818, D13S317, D7S820, TH01, TPOX, CSF1PO) in two urban populations from La Paz and Santa Cruz, Bolivia, and compares these frequencies with data published from other populations.

\section{SUBJECTS AND METHODS}

Blood samples were collected from 176 unrelated Bolivian individuals from the cities of La Paz $(\mathrm{N}=176)$ and Santa Cruz $(\mathrm{N}=84)$, Bolivia, who asked for paternity testing from 1999 to 2005. Genomic DNA was obtained by the organic extraction method described by Comey et al. (1994). The vWA, CSF1PO, TPOX, and TH01 STRs analyzed before November 2000 were amplified using $1 \mathrm{ng}$ DNA for each polymerase chain reaction, and the fragments were electrophoresed on 6\% acrylamide gels and silver stained (GenePrint ${ }^{\mathrm{TM}}$ STR systems, 1997). The D3S1358, vWA, FGA, D8S1179, D21S11, D18S51, D5S818, D13S317, D7S820, TH01, TPOX, and CSF1PO loci studied after November 2000 were amplified using the AmpFl STR $^{\circledR}$ Profiler Plus ${ }^{\mathrm{TM}}$ kit from Applied Biosystems. DNA fragments were electrophoresed on the ABI PRISM 310 genetic analyzer from Applied Biosystems-Perkin Elmer using the POP 4310 GA polymer. Data were obtained using the GeneScan 3.1, and Genotyper software from Applied Biosystems.

The allele frequencies were estimated by simple counting and the unbiased heterozygosity was calculated (Nei, 1992; Edwards et al., 1992). Both gene frequencies (expected according the Hardy-Weinberg equilibrium and those actually observed) were compared using the homogeneity $\chi^{2}$ test (Chakraborty et al., 1991; Nei, 1992). Comparisons of gene frequencies among populations were made by means of the non-parametric method described by Roff and Bentzen et al. (1989).

\section{RESULTS AND DISCUSSION}

The gene frequencies estimated for the La Paz and Santa Cruz samples were simi$\operatorname{lar}(\mathrm{P}>0.05)$ and therefore pooled in Table 1. Every locus analyzed fit the Hardy-Weinberg equilibrium $(\mathrm{P}>0.05)$. The lowest observed heterozygosity was 0.63 for the TPOX locus, while the highest observed heterozygosity was for the FGA locus (0.92). These gene frequencies are significantly different from those described for Chile (Figueroa et al., 2000; Cifuentes et al., 2002) and the United States Hispanic-Americans published by GenePrint ${ }^{\text {TM }}$ STR systems (1997) for every locus $(\mathrm{P}<0.05$ for all of them) with the exceptions of D3S1358, D18S51, TPOX, and CSF1PO loci. 
Table 1. Gene frequencies (G.F.) for the D3S1358, vWA, FGA, D8S1179, D21S11, D18S51, D5S818, D13S317, D7S820, TH01, TPOX, and CSF1PO loci in two samples from Bolivia.

\begin{tabular}{|c|c|c|c|c|c|c|}
\hline Loci & D3S1358 & vWA & FGA & D8S1179 & D21S11 & D18S51 \\
\hline Homozygotes & 31 & 44 & 8 & 18 & 14 & 13 \\
\hline Heterozygotes & 74 & 132 & 98 & 95 & 93 & 81 \\
\hline Total & 105 & 176 & 106 & 113 & 107 & 94 \\
\hline$\underline{\text { Allele }}$ & G.F. & G.F. & G.F. & G.F. & G.F. & G.F. \\
\hline \multicolumn{7}{|l|}{$\overline{6}$} \\
\hline \multicolumn{7}{|l|}{6.3} \\
\hline \multicolumn{7}{|l|}{7} \\
\hline \multicolumn{7}{|l|}{8} \\
\hline 9 & & & & 0.0044 & & 0.0053 \\
\hline 9.3 & & & & 0.0000 & & 0.0000 \\
\hline 10 & & & & 0.0752 & & 0.0160 \\
\hline 11 & & & & 0.0619 & & 0.0106 \\
\hline 12 & & & & 0.1327 & & 0.1436 \\
\hline 13 & 0.0048 & 0.0028 & & 0.3097 & & 0.1117 \\
\hline 14 & 0.0333 & 0.0426 & & 0.2257 & & 0.2021 \\
\hline 15 & 0.4143 & 0.0966 & & 0.1593 & & 0.1649 \\
\hline 16 & 0.3095 & 0.3352 & & 0.0265 & & 0.1223 \\
\hline 16.5 & 0.0000 & 0.0000 & & 0.0000 & & 0.0000 \\
\hline 17 & 0.1524 & 0.3494 & & 0.0044 & & 0.0957 \\
\hline 18 & 0.0762 & 0.1080 & 0.0094 & & & 0.0798 \\
\hline 19 & 0.0048 & 0.0597 & 0.1698 & & & 0.0213 \\
\hline 20 & 0.0000 & 0.0028 & 0.1038 & & & 0.0053 \\
\hline 21 & 0.0048 & 0.0000 & 0.0566 & & & 0.0213 \\
\hline 21.2 & & & 0.0000 & & & \\
\hline 22 & & & 0.0991 & & & \\
\hline 22.2 & & & 0.0047 & & & \\
\hline 23 & & & 0.1321 & & & \\
\hline 23.2 & & & 0.0000 & & & \\
\hline 24 & & & 0.1415 & & & \\
\hline 24.2 & & & 0.0000 & & & \\
\hline 25 & & & 0.1745 & & & \\
\hline 26 & & & 0.0849 & & & \\
\hline 27 & & & 0.0236 & & 0.0140 & \\
\hline 28 & & & & & 0.0514 & \\
\hline 29 & & & & & 0.1869 & \\
\hline 30 & & & & & 0.2103 & \\
\hline 30.2 & & & & & 0.0421 & \\
\hline 31 & & & & & 0.0794 & \\
\hline 31.2 & & & & & 0.1215 & \\
\hline 32 & & & & & 0.0047 & \\
\hline 32.2 & & & & & 0.2056 & \\
\hline 33 & & & & & 0.0000 & \\
\hline 33.2 & & & & & 0.0841 & \\
\hline 34.2 & & & & & 0.0000 & \\
\hline Total & 1.0000 & 1.0000 & 1.0000 & 1.0000 & 1.0000 & 1.0000 \\
\hline $\begin{array}{l}\text { Observed } \\
\text { heterozygosity }\end{array}$ & 0.7 & 0.75 & 0.92 & 0.84 & 0.87 & 0.86 \\
\hline Gene diversity & 0.7 & 0.74 & 0.87 & 0.80 & 0.86 & 0.87 \\
\hline
\end{tabular}


Table 1. Continued.

\begin{tabular}{|c|c|c|c|c|c|c|}
\hline Loci & D5S818 & D13S317 & D7S820 & TH01 & TPOX & CSF1PO \\
\hline Homozygotes & 22 & 13 & 18 & 25 & 33 & 22 \\
\hline Heterozygotes & 83 & 92 & 83 & 60 & 57 & 62 \\
\hline Total & 105 & 105 & 101 & 85 & 90 & 84 \\
\hline Allele & G.F. & G.F. & G.F. & G.F. & $\underline{\text { G.F. }}$ & G.F. \\
\hline 6 & & & & 0.2590 & 0.0111 & \\
\hline 6.3 & & & & 0.0000 & 0.0000 & \\
\hline 7 & 0.1571 & 0.0084 & 0.0050 & 0.4000 & 0.0167 & \\
\hline 8 & 0.0095 & 0.0714 & 0.0495 & 0.0471 & 0.5440 & \\
\hline 9 & 0.0762 & 0.2333 & 0.0644 & 0.0647 & 0.0611 & 0.0179 \\
\hline 9.3 & 0.0000 & 0.0000 & 0.0000 & 0.2353 & 0.0111 & 0.0000 \\
\hline 10 & 0.0238 & 0.0857 & 0.3218 & & 0.0333 & 0.2143 \\
\hline 11 & 0.4190 & 0.1857 & 0.3119 & & 0.1944 & 0.2738 \\
\hline 12 & 0.2190 & 0.1714 & 0.2079 & & 0.1278 & 0.3988 \\
\hline 13 & 0.0857 & 0.1333 & 0.0396 & & & 0.0714 \\
\hline 14 & 0.0095 & 0.1095 & & & & 0.0179 \\
\hline 15 & & 0.0048 & & & & 0.0060 \\
\hline \multicolumn{7}{|l|}{16} \\
\hline \multicolumn{7}{|l|}{16.5} \\
\hline \multicolumn{7}{|l|}{17} \\
\hline \multicolumn{7}{|l|}{18} \\
\hline \multicolumn{7}{|l|}{19} \\
\hline \multicolumn{7}{|l|}{20} \\
\hline \multicolumn{7}{|l|}{21} \\
\hline \multicolumn{7}{|l|}{21.2} \\
\hline \multicolumn{7}{|l|}{22} \\
\hline \multicolumn{7}{|l|}{22.2} \\
\hline \multicolumn{7}{|l|}{23} \\
\hline \multicolumn{7}{|l|}{23.2} \\
\hline \multicolumn{7}{|l|}{24} \\
\hline \multicolumn{7}{|l|}{24.2} \\
\hline \multicolumn{7}{|l|}{25} \\
\hline \multicolumn{7}{|l|}{26} \\
\hline \multicolumn{7}{|l|}{27} \\
\hline \multicolumn{7}{|l|}{28} \\
\hline \multicolumn{7}{|l|}{29} \\
\hline \multicolumn{7}{|l|}{30} \\
\hline \multicolumn{7}{|l|}{30.2} \\
\hline 31 & & & & & & \\
\hline 31.2 & & & & & & \\
\hline 32 & & & & & & \\
\hline 32.2 & & & & & & \\
\hline 33 & & & & & & \\
\hline 33.2 & & & & & & \\
\hline 34.2 & & & & & & \\
\hline 35 & & & & & & \\
\hline Total & 1.0000 & 1.0000 & 1.0000 & 1.0000 & 1.0000 & 1.0000 \\
\hline $\begin{array}{l}\text { Observed } \\
\text { heterozygosity }\end{array}$ & 0.79 & 0.88 & 0.82 & 0.71 & 0.63 & 0.74 \\
\hline Gene diversity & 0.74 & 0.84 & 0.75 & 0.71 & 0.64 & 0.71 \\
\hline
\end{tabular}




\section{REFERENCES}

Chakraborty R, Fonage M, Guegue R and Boerwinkle E (1991). Population genetics of hypervariable loci: Analysis of PCR-based VNTR polymorphism within a population. In: DNA fingerprinting: approaches and applications (Burke T, Dolf G, Jeffreys AJ and Wolff R, eds.). Birkhauser Verlag, Berlin, 127-143.

Cifuentes L, Acuna M and Sepulveda D (2002). Allele frequencies for six STR in a Chilean population. J. Forensic Sci. 47: 904-905.

Comey CT, Koons BW, Presley KW, Smerick JB, et al. (1994). DNA extraction strategies for amplified fragment length polymorphism analysis. J. Forensic Sci. 39: 1254-1269.

Edwards A, Hammond HA, Jin L, Caskey CT, et al. (1992). Genetic variation at five trimeric and tetrameric tandem repeat loci in four human population groups. Genomics 12: 241-253.

Figueroa CC, Acuna M and Cifuentes L (2000). Gene frequencies for six STR loci in a Chilean population of mixed ancestry. J. Forensic Sci. 45: 742-743.

GenePrint ${ }^{\mathrm{TM}}$ STR systems (1997). Technical manual. Promega Corporation, Madison.

Nei M (1992). Estimation of average heterozygosity and genetic distance from a small number of individuals. Genetics 89: 583-590.

Roff DA and Bentzen P (1989). The statistical analysis of mitochondrial DNA polymorphisms: chi 2 and the problem of small samples. Mol. Biol. Evol. 6: 539-545. 